\title{
The Canadian Chronic Disease Surveillance System: A Distributed Surveillance Model
}

\author{
Lisa Lix ${ }^{* 1,2}$ and Kim Reimer ${ }^{3}$ \\ ${ }^{1}$ Community Health Sciences, University of Manitoba, Winnipeg, MB, Canada; ${ }^{2}$ George and Fay Yee Centre for Healthcare \\ Innovation, Winnipeg, MB, Canada; ${ }^{3}$ British Columbia Ministry of Healthy Living and Sport, Victoria, BC, Canada
}

\section{Objective}

To describe the process, benefits, and challenges of implementing a distributed model for chronic disease surveillance across thirteen Canadian jurisdictions.

\section{Introduction}

The Public Health Agency of Canada (PHAC) established the Canadian Chronic Disease Surveillance System (CCDSS) in 2009 to facilitate national estimates of chronic disease prevalence, incidence, and health outcomes. The CCDSS uses population-based linked health administrative databases from all provinces/territories $(\mathrm{P} / \mathrm{Ts})$ and a distributed analytic protocol to produce standardized disease estimates.

\section{Methods}

The CCDSS is founded on deterministic linkage of three administrative health databases in each Canadian P/T: health insurance registration files, physician billing claims, and hospital discharge abstracts. Data on all residents who are eligible for provincial or territorial health insurance (about $97 \%$ of the Canadian population) are captured in the health insurance registration files. Thus, the CCDSS coverage is near-universal. Disease case definitions are developed by expert Working Groups after literature reviews are completed and validation studies are undertaken. Feasibility studies are initiated in selected $\mathrm{P} / \mathrm{Ts}$ to identify challenges when implementing the disease case definitions. Analytic code developed by PHAC is then distributed to all $\mathrm{P} / \mathrm{Ts}$. Data quality surveys are routinely conducted to identify database characteristics that may bias disease estimates over time or across $\mathrm{P} / \mathrm{Ts}$ or affect implementation of the analytic code. The summary data produced in each $\mathrm{P} / \mathrm{T}$ are approved by Scientific Committee and Technical Committee members and then submitted to PHAC for further analysis and reporting.

\section{Results}

National surveillance or feasibility studies are currently ongoing for diabetes, hypertension, selected mental illnesses, chronic respiratory diseases, heart disease, neurological conditions, musculoskeletal conditions, and stroke. The advantages of the distributed analytic protocol are (Figure 1): (a) changes in methodology can be easily made, and (b) technical expertise to implement the methodology is not required in each $\mathrm{P} / \mathrm{T}$. Challenges in the use of the distributed analytic protocol are: (a) heterogeneity in healthcare databases across $\mathrm{P} / \mathrm{Ts}$ and over time, (b) the requirement that each $\mathrm{P} / \mathrm{T}$ use the minimum set of data elements common to all jurisdictions when producing disease estimates, and (c) balancing disclosure guidelines to ensure data confidentiality with comprehensive reporting. Additional challenges, which include incomplete data capture for some databases and poor measurement validity of disease diagnosis codes for some chronic conditions, must be continually addressed to ensure the scientific rigor of the CCDSS methodology.

\section{Conclusions}

The CCDSS distributed analytic protocol offers one model for national chronic disease surveillance that has been successfully implemented and sustained by PHAC and its P/T partners. Many lessons have been learned about national chronic disease surveillance involving jurisdictions that are heterogeneous with respect to healthcare databases, expertise, and population characteristics.

\begin{tabular}{|c|c|c|}
\hline Features & Benefits & Challenges \\
\hline $\begin{array}{l}\text { National and subpopulation (ie., } \\
\text { age, sex, geographyy prevalance } \\
\text { and incidence estimates are } \\
\text { produced }\end{array}$ & $\begin{array}{l}\text { Changes in disease case } \\
\text { assertainment and estimation } \\
\text { methods can be easily made by } \\
\text { PHAC staff }\end{array}$ & $\begin{array}{l}\text { Heterogenenity in administrative } \\
\text { health databases may affect } \\
\text { accuracy of prevalence cstmates }\end{array}$ \\
\hline $\begin{array}{l}\text { Longitudinal estimates of } \\
\text { prevalence and incidence enable } \\
\text { comparisons across time }\end{array}$ & \multirow{2}{*}{$\begin{array}{l}\text { Technical expertise to mplement } \\
\text { the methodology is not required in } \\
\text { each PT }\end{array}$} & \multirow{2}{*}{$\begin{array}{l}\text { The CCDSS relies on the minimum } \\
\text { set of data elements common to all } \\
\text { PTIs to produce disease estimates }\end{array}$} \\
\hline $\begin{array}{l}\text { The CCDSS respects the data } \\
\text { custodial responsibilities of the } \\
\text { P T/s }\end{array}$ & & \\
\hline $\begin{array}{l}\text { SAS code to produce disease } \\
\text { estimates is developed by PHAC } \\
\text { staff }\end{array}$ & $\begin{array}{l}\text { Methodology to intiate } \\
\text { surveillance of new diseases can be } \\
\text { developed quickly for the P PTs }\end{array}$ & $\begin{array}{l}\text { Disclosure rules spectic to each } \\
\text { PT must be adhered to in order to } \\
\text { ensure data corfidentiality }\end{array}$ \\
\hline $\begin{array}{l}\text { Routinc data quality surveys aid in } \\
\text { interpretation of estimates }\end{array}$ & \multirow{2}{*}{$\begin{array}{l}\text { Report preparation and data } \\
\text { analyses are supported by PHAC } \\
\text { experts }\end{array}$} & \multirow{2}{*}{$\begin{array}{l}\text { There are ongoing concerns about } \\
\text { the quality (e.g., completeness \& } \\
\text { accuracy) of the administrative } \\
\text { heathcare databases }\end{array}$} \\
\hline $\begin{array}{l}\text { P/T experts contrbute their } \\
\text { knowledge on an ongoing basis }\end{array}$ & & \\
\hline
\end{tabular}

Figure 1. Features, Benefits, and Challenges of a Distributed Model for Disease Surveillance in Canada

\section{Keywords}

national surveillance; distributed analysis model; administrative data

\section{Acknowledgments}

This research was made possible through collaborations between the Public Health Agency of Canada (PHAC) and the provincial/territorial governments of British Columbia, Manitoba, Ontario, Quebec, New Brunswick, Nova Scotia, Newfoundland and Labrador, Yukon, Northwest Territories, and Nunavut.

*Lisa Lix

E-mail: lisa.lix@umanitoba.ca 